\title{
Impact des plantations de palmier à huile (Elaeis guineensis Jacq.), conditions environnementales et du temps sur la structure des communautés d'Acariens du sol de la station de La Mé, Côte d'Ivoire
}

\author{
Jean-Luc D.S. AHUI', Julien K. N'DRI1,2, Ettien F. EDOUKOU², Joseph G. YEO1 \\ 1 Unité de Formation et de Recherche (UFR) des Sciences de la Nature, Université Nangui Abrogoua, 02 BP 801 Abidjan \\ 02, Côte d'Ivoire. E-mail : ahuidieudonne@gmail.com; ndri_kk@yahoo.fr; gnenis@gmail.com \\ ${ }^{2}$ Centre de Recherche en Écologie, 08 BP 109 Abidjan 08, Côte d'Ivoire. E-mail : edflavien@yahoo.fr; ndri_jk@yahoo.fr
}

Original submitted in on 31st May 2018. Published online at www.m.elewa.org on 30 September 2018 http://dx.doi.org/10.4314/jab.v129i1.12

\begin{abstract}
RESUME
Objectif : L'étude réalisée au sein de la station de La Mé consistait à évaluer les différentes modifications de la structure des communautés d'Acariens du sol observés à travers une chronoséquence de palmier à huile. Méthodologie et résultats : En pratique, quatre modes d'utilisation du sol sont sélectionnées (forêt secondaire, plantations de palmiers âgées de 13, 20 et 39 ans), où chaque mode d'utilisation du sol est répliqué trois fois. Les carottes de sol $(0-10 \mathrm{~cm})$ pour l'extraction des Acariens sont prélevées à l'aide d'une tarière de $5 \mathrm{~cm}$ de diamètre suivant un transect de $50 \mathrm{~m}$. Cinq carottes de sol sont prélevées par transect, soit un total de 60 carottes de sol pour l'ensemble des 12 parcelles d'échantillonnages. Les résultats révèlent que la densité des Acariens (-88\% et $-48 \%)$, la richesse spécifique $(-64 \%$ et $-21 \%)$ et la diversité $(-64 \%$ et $-8 \%)$ ont diminué, respectivement, 13 ans et 39 ans après la conversion de la forêt secondaire en plantations de palmiers. Au total, 68 espèces d'Acariens ont été répertoriées le long des modes d'utilisation du sol. L'abondance relative des Oribates augmente avec l'âge croissant des plantations de palmiers tandis que la tendance inverse est observée avec les Gamasides. Les espèces rares et les espèces eudominantes caractérisent la forêt secondaire, pendant que les espèces dominantes et sous-dominantes prédominent dans les plantations de palmiers âgées de 13, 20 et 39 ans. L'étude révèle également une forte dissemblance dans la composition spécifique des communautés d'Acariens observées le long de la chronoséquence de palmiers. L'écosystème forestier est plus favorable au développement des Acariens, cependant une tendance à la recolonisation des plantations serait plus importante au fur et à mesure que les plantations de palmiers vieillissent.

Conclusion et application des résultats : Après 39 ans de conversion de la forêt secondaire en plantations de palmiers, le processus de restauration de la qualité du sol n'a pas favorisé un changement significatif des composantes biologique du sol. Néanmoins, cette investigation souligne une amélioration de la qualité biologique du sol avec le temps, et qui se caractérise par une augmentation de la densité des Acariens $(+336 \%)$, richesse spécifique $(+121 \%)$ et de la diversité $(+153 \%)$, respectivement dans les plantations âgées de 39 ans comparées à celles de 13 ans.
\end{abstract}


Ahui et al., J. Appl. Biosci. 2018 Impact des plantations de palmier à huile conditions environnementales et du temps sur la structure des communautés d'Acariens du sol de la station de La Mé, Côte d'Ivoire

Mots clés : Abondance, Diversité, Structure des communautés, Acariens du sol, Plantations de palmier à huile, Chronoséquence.

\section{ABSTRACT}

Impact of Oil palm (Elaeis guineensis Jacq.) plantations, environmental conditions and time on soil mite communities structure of La Mé station, Côte d'Ivoire

Objective: The study undertaken in La Mé station consisted to evaluate the different modifications of soil mite communities structure observed across the Oil palm chronosequence.

Methodology and results : In practice, four land use types were selected (secondary forest, 13-, 20-, and 39year-old Oil palm plantations), where each land use type was replicated three time. The soil cores $(0-10 \mathrm{~cm}$, soil depth) for mite's extraction were taken during the wet period with a steel corer $(\varnothing 5 \mathrm{~cm})$ following a $50 \mathrm{~m}$ transect. Five soil cores were taken per transect, whether a total of 60 soil cores for the 12 sampling areas. The results showed that the mites density (-88\% and $-48 \%)$, species richness (-64\% and $-21 \%)$, and diversity $(-64 \%$ and $-8 \%$ ) decreased, respectively, 13 years and 39 years after the secondary forest conversion into Oil palm plantations. In total, 68 species of mites were recorded through the land use types. The relative abundance of Oribatida increased with the increasing age of the Oil palm plantations whereas the reverse trend is observed with the Gamasida. The rare species and eudominant species characterized the secondary forest, while the dominant and subdominant species preponderate in 13-, 20-, and 39-year-old Oil palm plantations. The study indicates a high dissemblance in specific composition of the mite communities across the Oil palm chronosequence. The forest ecosystem is more favorable to the development of mites; however, a trend towards recolonization of the plantations would be more important with the aging of the Oil palm plantations. Conclusion and application of results : After 39 years of secondary forest conversion into Oil palm plantations, the process of soil quality restoration did not favor a significant change in soil biological components. Nevertheless, this investigation highlights an improvement in the soil biological quality over time, and characterized by an increase in the density of mites (+336\%), species richness $(+121 \%)$ and diversity $(+153 \%)$, respectively, in the 39-year-old plantations compared to the 13-year-old plantations.

Keywords : Abundance, Diversity, Communities structure, Soil mites, Oil palm plantations, Chronosequence.

\section{INTRODUCTION}

Les forêts jouent un rôle important dans le maintien de la biodiversité et l'atténuation du changement climatique (Felton et al., 2016). Toutefois, la transformation des forêts tropicales et l'intensification des modes d'utilisation des terres entraînent une érosion des stocks de carbone et des pertes substantielles en termes de biodiversité et de fonctions écosystémiques (Drescher et al., 2016). La récente évaluation faite par la FAO indique qu'entre 1990 et 2015 , la surface forestière mondiale a diminué de $3 \%$, passant de 4128 millions d'hectares à 3999 millions (Keenan et al., 2015). Cette réduction du couvert forestier mondiale est en partie due à la culture du palmier à huile (Conti, 2015; Tao et al., 2016). En Afrique, le palmier à huile (Elaeis guineensis Jacq.) est cultivé sur une superficie totale de 16 millions d'hectares (Carron et al., 2015). 20 à $25 \%$ des émissions du carbone mondial proviendrait de la déforestation (IPCC, 2001). Les forêts abritent de nombreuses espèces animales et végétales, et produisent des biens et services éco-systémiques indispensables au maintien de la vie sur terre (Thompson, 2011 ; Scriven et al., 2015). Cependant la perturbation liée à la déforestation abusive pourrait contribuer à long terme à une érosion des organismes du sol (Čuchta et Starý, 2015) et impacter négativement la capacité productive de cet écosystème (Thompson, 2011 ; Gérard, 2016). En Côte d'Ivoire, le couvert végétal a connu une forte régression depuis l'indépendance (Brou Yao et al., 2005). En effet, l'expansion de certaines cultures pérennes ont contribué à la transformation des forêts en plantations et au déséquilibre des écosystèmes naturelles (Kangah et Koli-Bi, 2015). Toutefois, 27\% du Produit Intérieur Brut (PIB) proviendrait du secteur agricole et rapporterait à l'économie 
ivoirienne $40 \%$ des recettes d'exportation (Cheyns et al., 2000 ; Sangaré et al., 2009). Spécifiquement, la production de l'huile de palme représenterait $3,13 \%$ du Produit Intérieur Brut (Palmafrique, 2017). Le problème de la réconciliation entre intérêts économiques et durabilité écologique en agriculture tropicale a toujours été une préoccupation de la part de la communauté internationale (Drescher et al., 2016). Localement, la culture du palmier à huile a particulièrement connu une expansion assez remarquable depuis les années 60 suite au vaste programme de développement de plants de palmiers à huile sélectionnés, et initié à l'époque par le gouvernement ivoirien. Cette expansion était marquée par la conversion d'environ 250000 hectares de forêt en plantations industrielles et villageoises (Carrère, 2010). Cependant, ces dernières années, le problème de la dégradation des sols et de l'environnement consécutif à la conversion des forêts en agrosystèmes demeure une préoccupation universelle (Koh et Wilcove, 2008 ; Gérard, 2016). Les activités agricoles telles que le désherbage, le labour, le brûlage, l'application de pesticides et la collecte après récolte des résidus végétaux modifient potentiellement les propriétés physico-chimiques et biologiques du sol (Gbarakoro et Zabbey, 2013). Les sols forestiers sont généralement caractérisés par une communauté biologique abondante et diversifiée (Barrios, 2007 ; Gbarakoro et Zabbey, 2013). Toutefois, avec la mise en place d'un système de gestion durable, les agrosystèmes peuvent fournir des conditions favorables pour la biodiversité du sol (Conti, 2015 ; Tao et al., 2016). La plupart des travaux de recherche mettant en exergue l'impact des agrosystèmes sur les organismes du sol sont

\section{MATERIEL ET METHODES}

Site d'étude : L'étude a été conduite en Août-Septembre 2017 dans la région de La Mé, précisément à la station de recherche CNRA de La Mé $\left(5^{\circ} 26^{\prime} \mathrm{N}, 3^{\circ} 50^{\prime} \mathrm{W}\right)$ située à $30 \mathrm{~km}$ au Nord-Est d'Abidjan. Le climat est de type équatorial, caractérisé par quatre saisons distinctes : une grande saison de pluie d'Avril à Juillet, une petite saison sèche en Août, une petite saison de pluie de Septembre à Novembre et une grande saison sèche de Décembre à beaucoup portés sur la macrofaune malgré la grande diversité des microarthropodes du sol (Barrios, 2007). Présent dans tous les compartiments de la biosphère (Lorber, 2017), les Acariens du sol jouent un rôle écologique important dans le fonctionnement des écosystèmes (Coleman et al., 2004). Ils sont très actifs dans le maintien de la fertilité du sol grâce à leurs activités régulatrices de décomposition et de renouvellement des nutriments (Acharya et al., 2014). Cependant, étant caractérisés par une très faible capacité de dispersion (Berthet, 1964), leur abondance et leur diversité sont considérablement affectés par le mode d'utilisation des terres agricoles (Bedano et Ruf, 2006; Singh et Ray, 2015), particulièrement pour les espèces ayant un cycle de vie supérieur à une année (Behan-Pelletier, 1999). Excepté l'investigation réalisée par N'Dri et al. (2017) à travers une chronoséquence de plantations d'hévéa, aucune étude ne fait référence à l'impact des plantations du palmier à huile et du temps sur l'abondance et la diversité des Acariens du sol en Côte d'Ivoire. La présente étude ambitionne d'évaluer les différentes modifications de la structure des communautés d'Acariens du sol observés à travers une chronoséquence de palmier à huile. Spécifiquement, il s'agira d'étudier l'abondance, la diversité ainsi que la structure des Acariens du sol. L'hypothèse qui soutient cette étude est que l'abondance et la diversité des Acariens du sol augmentent avec l'âge croissant des plantations de palmier à huile après une baisse relative à la conversion de la forêt secondaire. En d'autre terme, nous nous attendions qu'avec le temps la structure des communautés d'Acariens dans les plantations de palmier à huile pourrait atteindre les conditions similaires à celles observées en forêt secondaire.

Mars. La pluviométrie moyenne mensuelle varie de 18 $\mathrm{mm}$ en Janvier à $486 \mathrm{~mm}$ en Juin avec un total annuel de $1915 \mathrm{~mm}$. La température moyenne mensuelle oscille entre $25^{\circ} \mathrm{C}$ en Août à $28^{\circ} \mathrm{C}$ en Février et Mars avec une moyenne annuelle de $27^{\circ} \mathrm{C}$. Les forêts de type ombrophile caractérisent la zone d'étude. Les espèces ligneuses Turraeanthus africanus et Heisteria parvifolia sont les plus dominantes (Traoré et Péné, 2009). Ces 
formations forestières naturelles se sont fortement dégradées depuis l'indépendance (Brou Yao et al., 2005). En effet, une grande partie de la forêt a pratiquement fait place à des exploitations agricoles dominées par le palmier à huile. Elaeis guineensis est l'espèce la plus cultivée. Originaire de la zone intertropicale humide d'Afrique (Jacquemard, 2011), la culture du palmier à huile s'est développée sans interruption jusqu'à présent dans la partie sud du pays (Jannot, 2010). Les sols sont de type ferralitique. Les textures sableuses et argilosableuses caractérisent les horizons de surface. Profonds, meubles et sans éléments grossiers, ces sols sont filtrants, légers et perméables à l'eau et à l'air. La culture du palmier à huile nécessite un sol meuble avec un $\mathrm{pH}$ compris entre 3,5 et 5,5 sur au moins $1 \mathrm{~m}$ de profondeur. Le palmier à huile croit de façon optimale sur des terrains plats ou à faibles pentes (0 à $12 \%$ ). II s'adapte aux textures sablo-argileuses et limonoargileuses (Paramananthan, 2013).
Choix des parcelles d'études : Afin de mieux apprécier la réponse des communautés d'Acariens du sol relative à la conversion des forêts secondaires en plantations de palmier à huile, quatre modes d'utilisation du sol sont sélectionnées (forêt secondaire, plantations de palmiers âgées de 13,20 et 39 ans), où chaque mode d'utilisation du sol est répliqué trois fois. II s'agit d'une approche dite de "chronoséquence » qui a pour avantage de tenir compte de l'impact de la dynamique spatio-temporelle des plantations de palmiers sur les variables étudiées (Walker et al., 2010). L'historique des parcelles d'études (Tableau 1) relate que les plantations de palmiers sont issues de la conversion (i) directe de la forêt primaire, ou (ii) indirecte, après une rotation culturale suivie parfois d'une période de jachère. Du point de vue économique, les âges 13 et 20 ans désignent la période de stabilisation de production tandis que celle de 39 ans désigne la période de décroissance de la production liée aux mortalités et aux difficultés de récolte (Rafflegeau, 2008).

Tableau 1 : Description des différents modes d'utilisation du sol

\begin{tabular}{c|c|c|c|c}
\hline Mode d'utilisation du sol & Age (années) & Type de sol & Texture du sol & Précédent cultural \\
\hline Palmier & 13 & Ferralitique & Sableuse & Forêt primaire- vieille palmeraie \\
Palmier & 13 & Ferralitique & Sableuse & Forêt primaire- vieille palmeraie \\
Palmier & 13 & Ferralitique & Argilo sableuse & Forêt primaire- vieille palmeraie \\
Palmier & 20 & Ferralitique & Sableuse & Forêt primaire- vieille palmeraie \\
Palmier & 20 & Ferralitique & Sableuse & Forêt primaire- vieille palmeraie \\
Palmier & 20 & Ferralitique & Argilo sableuse & Forêt primaire- vieille palmeraie \\
Palmier & 39 & Ferralitique & Argilo sableuse & Forêt primaire-Maïs-Jachère \\
Palmier & 39 & Ferralitique & Argilo sableuse & Forêt primaire-Manioc-Jachère \\
Palmier & 39 & Ferralitique & Argilo sableuse & Forêt primaire \\
Forêt secondaire & 150 & Ferralitique & Argilo sableuse & Forêt primaire \\
Forêt secondaire & 150 & Ferralitique & Argilo sableuse & Forêt primaire \\
Forêt secondaire & 150 & Ferralitique & Argilo sableuse & Forêt primaire \\
\hline
\end{tabular}

Caractérisation des propriétés physico-chimiques du sol: Les propriétés physiques du sol ne diffèrent pas significativement à travers les modes d'utilisation du sol. La densité apparente varie entre $0,91 \pm 0,05 \mathrm{~g} \cdot \mathrm{cm}^{-3}$ (forêts secondaires) et 1,20 $\pm 0,06 \mathrm{~g}_{\mathrm{cm}} \mathrm{cm}^{-3}$ (plantations de palmiers âgées de 13 ans) tandis que la teneur en eau du sol oscille entre $10,95 \pm 0,82 \%$ (plantations de palmiers âgées de 13 ans) et 16,34 $\pm 2,20 \%$ (plantations de palmiers âgées de 20 ans). Contrairement aux propriétés physiques, les propriétés chimiques du sol diffèrent significativement à travers les modes d'utilisation du sol
(Tableau 2). Les sols sont généralement acides. A l'exception du carbone accumulé annuellement et du pH, les autres propriétés chimiques du sol présentent des valeurs similaires (Carbone organique du sol : 19,55 \pm $1,33 \mathrm{~g} \cdot \mathrm{kg}^{-1}$ sol vs. $19,55 \pm 2,70 \mathrm{~g} \cdot \mathrm{kg}^{-1}$ sol ; Matière organique du sol : $33,23 \pm 2,27$ g.kg-1 sol vs. $33,23 \pm 4,59$ g.kg-1 sol ; Stock de carbone: $17,64 \pm 1,33$ t.ha-1 $^{-1}$ vs. 18,16 $\pm 2,73$ tha- $^{-1}$; Carbone séquestré : $64,70 \pm 4,90$ Teq $\mathrm{CO}_{2} \cdot$ ha $^{-1}$ vs. $66,58 \pm 10,01$ Teq $\mathrm{CO}_{2} \cdot$ ha $^{-1}$ ), respectivement sous la forêt secondaire et les plantations de palmiers âgées de 39 ans. 
Tableau 2 : Caractéristiques physico-chimiques du sol. FS-Forêts secondaires, P13-Plantations de palmiers âgées de 13 ans, P20-Plantations de palmiers âgées de 20 ans, P39-Plantations de palmiers âgées de 39 ans.

\begin{tabular}{|c|c|c|c|c|c|c|c|c|c|}
\hline Caractéristiques du sol & FS & & P13 & & P20 & & P39 & & \\
\hline Densité apparente $\left(\mathrm{g} \cdot \mathrm{cm}^{-3}\right)$ & 0,91 & $\pm 0,05^{a}$ & 1,20 & $\pm 0,06 \mathrm{a}$ & 1,07 & $\pm 0,06^{a}$ & 1,04 & $\pm 0,12^{\mathrm{a}}$ & 0,1080 \\
\hline Teneur en eau (\%) & 12,02 & $\pm 1,21 \mathrm{a}$ & 10,95 & $\pm 0,82^{a}$ & 16,34 & $\pm 2,20^{a}$ & 14,00 & $\pm 3,42^{\mathrm{a}}$ & 0,3250 \\
\hline $\begin{array}{l}\text { Carbone organique du sol } \\
\left(\mathrm{g} . \mathrm{kg}^{-1} \mathrm{sol}\right)\end{array}$ & 19,55 & $\pm 1,33^{a}$ & 11,42 & $\pm 0,53^{b}$ & 17,50 & $\pm 1,86 \mathrm{ab}$ & 19,55 & $\pm 2,70^{a}$ & 0,0086 \\
\hline $\begin{array}{l}\text { Matière organique du sol } \\
\left(\mathrm{g} . \mathrm{kg}^{-1} \mathrm{sol}\right)\end{array}$ & 33,23 & $\pm 2,27^{a}$ & 19,42 & $\pm 0,90^{b}$ & 29,75 & $\pm 3,16^{\mathrm{ab}}$ & 33,23 & $\pm 4,59^{a}$ & 0,0086 \\
\hline $\mathrm{pH}-\mathrm{H}_{2} \mathrm{O}$ & 5,00 & $\pm 0,15^{b}$ & 5,95 & $\pm 0,30^{a}$ & 4,91 & $\pm 0,07^{b}$ & 5,28 & $\pm \underset{b}{0,09 a}$ & 0,0009 \\
\hline $\begin{array}{l}\text { Carbone accumulé (t.ha- } \\
\text { 1.an-1) }\end{array}$ & 0,11 & $\pm 0,01 \mathrm{c}$ & 0,80 & $\pm 0,06^{a}$ & 0,82 & $\pm 0,10^{a}$ & 0,47 & $\pm 0,07 \mathrm{~b}$ & 0,0001 \\
\hline Stock de carbone (t.ha-1) & 17,64 & $\pm 1,33^{\mathrm{ab}}$ & 10,43 & $\pm 0,84^{b}$ & 16,33 & $\pm 2,18 \mathrm{ab}$ & 18,16 & $\pm 2,73^{a}$ & 0,0283 \\
\hline $\begin{array}{l}\text { Carbone séquestré (Teq } \\
\mathrm{CO}_{2} \cdot \text { ha-1}^{-1} \text { ) }\end{array}$ & 64,70 & $\pm 4,90 \mathrm{ab}$ & 38,24 & $\pm 3,08^{b}$ & 59,87 & $\pm 8,03 \mathrm{ab}$ & 66,58 & $\pm \underset{\mathrm{a}}{10,01}$ & 0,0283 \\
\hline
\end{tabular}

$\mathrm{N}=36$, Test d'Anova $1,{ }^{*} p<0,05 ;{ }^{* *} p<0,01 ;{ }^{* * *} p<0,001$. Au niveau de chaque ligne, les valeurs portant la même lettre en exposant ne sont pas statistiquement différentes au niveau $p=0,05$. (test de comparaison multiple de Tukey).

Prélèvement des carottes de sol : Les carottes de sol pour l'extraction des Acariens sont prélevées à $10 \mathrm{~cm}$ de profondeur du sol à l'aide d'une tarière de $5 \mathrm{~cm}$ de diamètre suivant un transect de $50 \mathrm{~m}$. Sur chaque parcelle d'étude, un seul transect est établie au centre de sorte à réduire les effets de bordure. Par transect, cinq points d'échantillonnages sont considérés avec une équidistance de $12,5 \mathrm{~m}$ entre deux points consécutifs. A chaque point d'échantillonnage, une carotte de sol est prélevée, soit un total de cinq carottes par transect. 60 carottes de sol sont prélevées sur l'ensemble des 12 parcelles d'échantillonnages. Minutieusement conservées dans des boîtes de collecte, les carottes de sol sont portées au laboratoire du Centre de Recherche en Écologie (CRE) pour l'extraction des Acariens.

Extraction et identification des Acariens du sol: L'extraction des Acariens est réalisée suivant la méthode de Berlese-Tullgren (Bedano et Ruf, 2006). Des ampoules de 40 Watts situées à $25 \mathrm{~cm}$ au-dessus des entonnoirs (1 ampoule pour 4 entonnoirs) ont permis de dessécher progressivement les carottes de sol contenues dans des tamis de $2 \mathrm{~mm}$ de maille pendant 10 jours. L'allumage des ampoules s'est fait systématiquement après la mise des carottes de sol au Berlese. Les
Acariens fuyant la chaleur, finissent par tomber dans les tubes de collecte contenant de l'alcool dilué à $70 \%$. Le dépouillement et le tri des Acariens sont réalisés dans des boîtes de Pétri sous une loupe binoculaire. Afin de permettre leur identification, des montages temporaires sont réalisés dans des lames à concavité contenant de l'acide lactique (85\%). Après leur fixation durant 48 heures, les Acariens sont identifiés sous un microscope optique muni d'une caméra. Les Acariens adultes sont identifiés aux niveaux des familles, genres et morphoespèces en utilisant les clés d'identifications et de descriptions fournies par Balogh et Balogh (1992), Krantz et Walter (2009), Walter et al., (2013). Toutefois la collection de référence locale a été consultée. Durant les séries d'identification, la classification moderne des Acariens proposée par Krantz et Walter (2009) a été appliquée.

Traitements des données : L'abondance des Acariens du sol est estimée par l'intermédiaire de la densité exprimée en nombre d'individus par mètre carré. La diversité des Acariens du sol est caractérisée par la richesse spécifique (nombre moyen d'espèces), l'indice de Shannon et l'équitabilité. L'abondance relative des groupes majeurs (Oribates et Gamasides) et des sous- 
groupes (Gamasina, Uropodina, Mixonomata, Desmonomata et Brachypylina) a permis d'étudier la structure des communautés d'Acariens observées le long de la chronoséquence de palmier à huile. La dominance relative des espèces d'Acariens est catégorisée suivant la classification de Yang et al. (2015). Suivant cette partition, les espèces eudominantes représentent plus de $30 \%$ du nombre total d'individus, les dominantes (10-30\%

$$
\operatorname{IST}=\left(\frac{\mathrm{H}}{\mathrm{h}}-1\right)^{1 / 2}
$$

Avec $\mathrm{h}$ le nombre de points d'échantillonnages où le taxon est observé; $\mathrm{H}$ le nombre total de points d'échantillonnages sur l'ensemble des modes d'utilisation du sol. L'indice de spécialisation de la communauté (ISC) est calculé comme étant la moyenne des IST. Afin d'apprécier l'efficacité de la méthode d'échantillonnage, le taux de recrutement des espèces au niveau de chaque mode d'utilisation du sol est évalué par l'intermédiaire de la richesse spécifique observée (Sobs) et de l'estimateur non paramétrique de la richesse spécifique attendue (Jacknife 1).

Analyses statistiques: Après vérification du test d'homogénéité (test de Levene) de l'abondance des Acariens, ainsi que celle des sous-groupes, les données

\section{RESULTATS}

Densité moyenne : La densité moyenne des Acariens observés varie de manière significative le long de la chronoséquence (Anova 1, $\mathrm{F}=3,79 ; \mathrm{P}=0,015$ ) et augmente avec l'âge croissant des plantations de palmiers (Figure 1). La densité moyenne observée en forêt secondaire $\left(6222 \pm 3386\right.$ ind. $\left.\mathrm{m}^{-2}\right)$ est quasiment le des individus), les sous-dominantes $(5-10 \%$ des individus), les mineures (1-5\% des individus) et les espèces rares (moins de $1 \%$ du nombre total des individus). Les espèces spécialistes et généralistes sont répertoriées le long de la chronoséquence de palmier à huile. L'indice de spécialisation taxonomique (Julliard et al., 2006) est déterminé par la formule suivante :

sont normalisées via la formule log $(x+1)$ comme recommandé par Gérard et Berthet (1966). L'analyse de la variance (Anova 1) au seuil de $5 \%$ a permis la comparaison de l'abondance, la diversité ainsi que la structure des communautés d'Acariens à travers les modes d'utilisation du sol. Une comparaison post-doc suivi d'un test HSD de Tukey a favorisé une comparaison deux à deux. L'impact des propriétés physico-chimiques du sol sur les caractéristiques des Acariens est étudié par l'intermédiaire de la corrélation de Spearman. Toutes ces analyses sont réalisées à l'aide du logiciel Statistica 7.1. Les estimateurs non paramétriques de la richesse spécifique sont calculés à partir de 500 simulations avec le logiciel Estimates 7.5 (Colwell, 2000).

double de celle enregistrée dans les plantations de palmiers âgées de 39 ans (3264 \pm 1556 ind. $\left.\mathrm{m}^{-2}\right)$. La densité des Acariens a diminué de $-88 \%$ et $-48 \%$, respectivement, 13 ans et 39 ans après la conversion de la forêt secondaire en plantations de palmiers. 


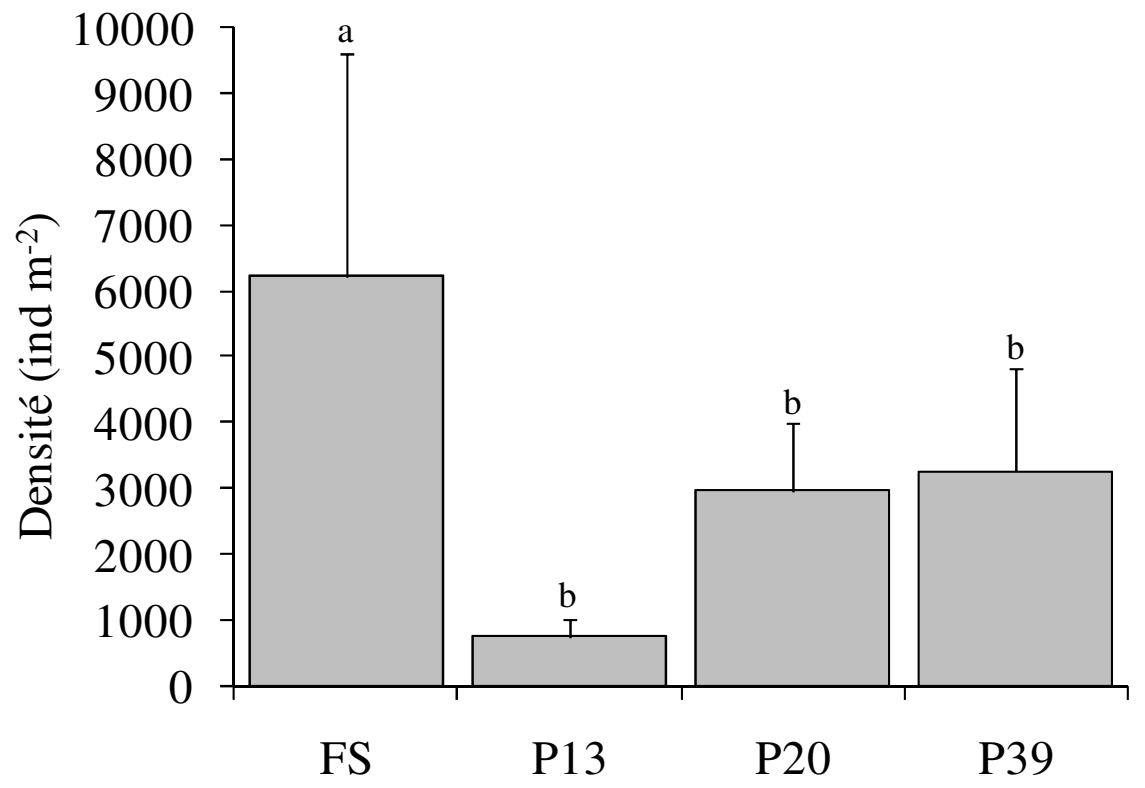

Mode d'utilisation du sol

Figure 1 : Densité moyenne des Acariens du sol. FS-Forêts secondaires, P13-Plantations de palmiers âgées de 13 ans, P20-Plantations de palmiers âgées de 20 ans, P39-Plantations de palmiers âgées de 39 ans. $N=60$, Test d'Anova 1 ; les histogrammes portant les mêmes lettres ne sont pas significativement différents au seuil de $5 \%$ (test de comparaison multiple de Tukey).

Richesse spécifique et diversité : Le nombre moyen d'espèces (Anova 1, $F=2,37 ; P=0,08$ ) et l'indice de Shannon (Anova $1, F=1,14 ; P=0,34$ ) ne diffèrent pas significativement à travers les modes d'utilisation du sol. Le nombre moyen d'espèces et l'indice de Shannon augmentent avec l'âge croissant des plantations de palmier à huile après une chute consécutive à la conversion des forêts secondaires en plantations (Tableau 3). Le nombre moyen d'espèces varie entre $1,07 \pm 0,33$ espèces (plantations de palmiers âgées de 13 ans) et 4,13 $\pm 1,12$ espèces (forêt secondaire). L'indice de Shannon enregistré en forêt secondaire $(0,83$ $\pm 0,24)$ est presque similaire à celui mesuré dans les plantations de palmiers âgées de 39 ans $(0,76 \pm 0,23)$. Les valeurs de l'équitabilité changent significativement d'un mode d'utilisation du sol à un autre (Anova 1, F = $6,39 ; P=0,0008)$. Les équitabilités enregistrées en forêt secondaire $(0,48 \pm 0,12)$ et dans les plantations de palmiers âgées de 39 ans $(0,51 \pm 0,13)$ sont quasiment identiques. Les plus faibles valeurs issues des plantations âgées de 13 ans $(0,06 \pm 0,06)$ et de 20 ans $(0,06 \pm 0,06)$ demeurent également similaires. La diversité des Acariens a baissé de $-64 \%$ et $-8 \%$, respectivement, 13 ans et 39 ans après la conversion de la forêt secondaire en plantations de palmiers.

Tableau 3 : Diversité des Acariens du sol. FS-Forêts secondaires, P13-Plantations de palmiers âgées de 13 ans, P20Plantations de palmiers âgées de 20 ans, P39-Plantations de palmiers âgées de 39 ans.

\begin{tabular}{|c|c|c|c|c|c|}
\hline & \multicolumn{4}{|c|}{ Mode d'utilisation du sol } & \multirow[b]{2}{*}{$P$ valeur } \\
\hline & FS & P13 & P20 & P39 & \\
\hline Richesse spécifique & $4,13 \pm 1,1^{a}$ & $1,06 \pm 0,33^{a}$ & $2,93 \pm 1,06^{a}$ & $3,4 \pm 1,05^{\mathrm{a}}$ & 0,080 \\
\hline Indice de Shannon & $0,83 \pm 0,23^{a}$ & $0,30 \pm 0,12^{\mathrm{a}}$ & $0,60 \pm 0,22^{a}$ & $0,76 \pm 0,22^{a}$ & 0,340 \\
\hline Equitabilité & $0,48 \pm 0,12^{\mathrm{a}}$ & $0,06 \pm 0,06^{b}$ & $0,06 \pm 0,06^{b}$ & $0,50 \pm 0,12^{\mathrm{a}}$ & $0,0008^{* * *}$ \\
\hline
\end{tabular}

$\mathrm{N}=60$, Test d'Anova $1,{ }^{* * *} \mathrm{p}<0,001$. Au niveau de chaque ligne, les valeurs portant la même lettre en exposant ne sont pas statistiquement différentes au niveau $p=0,05$. (test de comparaison multiple de Tukey). 


\section{Structure des communautés}

Acariens du sol: Au total, 68 espèces d'Acariens sont répertoriées le long des modes d'utilisation du sol. 39, 14, 32 et 31 espèces sont observées respectivement en forêt secondaire et dans les plantations de palmiers âgées de 13, 20 et 39 ans (Tableau 4). La richesse spécifique observée des Acariens a régressé de $-64 \%$ et $-21 \%$, respectivement, 13 ans et 39 ans après la conversion de la forêt secondaire en plantations de palmiers. L'indice de complémentarité de Sørensen (85\%) est plus grand entre la forêt secondaire et les plantations de palmiers âgées de 13 ans, et relativement faible (46\%) entre les plantations de palmiers âgées de 20 ans et 39 ans. Ce qui traduit une forte dissemblance dans la composition spécifique des communautés d'Acariens issues des différents modes d'utilisation du sol. Toutefois, l'estimateur non paramétrique Jacknife 1 indique que la richesse spécifique attendue pourrait varier entre 25 et 65 espèces, soit un taux de recrutement d'espèces oscillant entre $56 \%$ et $66 \%$. Les Gamasides ( $21 \%$ à $41 \%$ ) et les Oribates $(59 \%$ à $79 \%)$ constituent les deux grands groupes de la faune acarologique du sol (Figure 2). L'abondance relative des Oribates augmente avec l'âge croissant des plantations de palmiers tandis que la tendance inverse s'observe avec les Gamasides.

Tableau 4 : Taux de recrutement des espèces d'Acariens. FS-Forêts secondaires, P13-Plantations de palmiers âgées de 13 ans, P20-Plantations de palmiers âgées de 20 ans, P39-Plantations de palmiers âgées de 39 ans.

\begin{tabular}{l|c|c|c|c}
\hline & \multicolumn{4}{|c}{ Mode d'utilisation du sol } \\
\cline { 2 - 5 } & FS & P13 & P20 & P39 \\
\hline Richesse spécifique observée (Sob) & 39 & 14 & 32 & 31 \\
Richesse spécifique attendue (Jacknife 1) & 65 & 25 & 53 & 47 \\
Taux de recrutement (\%) & 60 & 56 & 60 & 66 \\
\hline
\end{tabular}

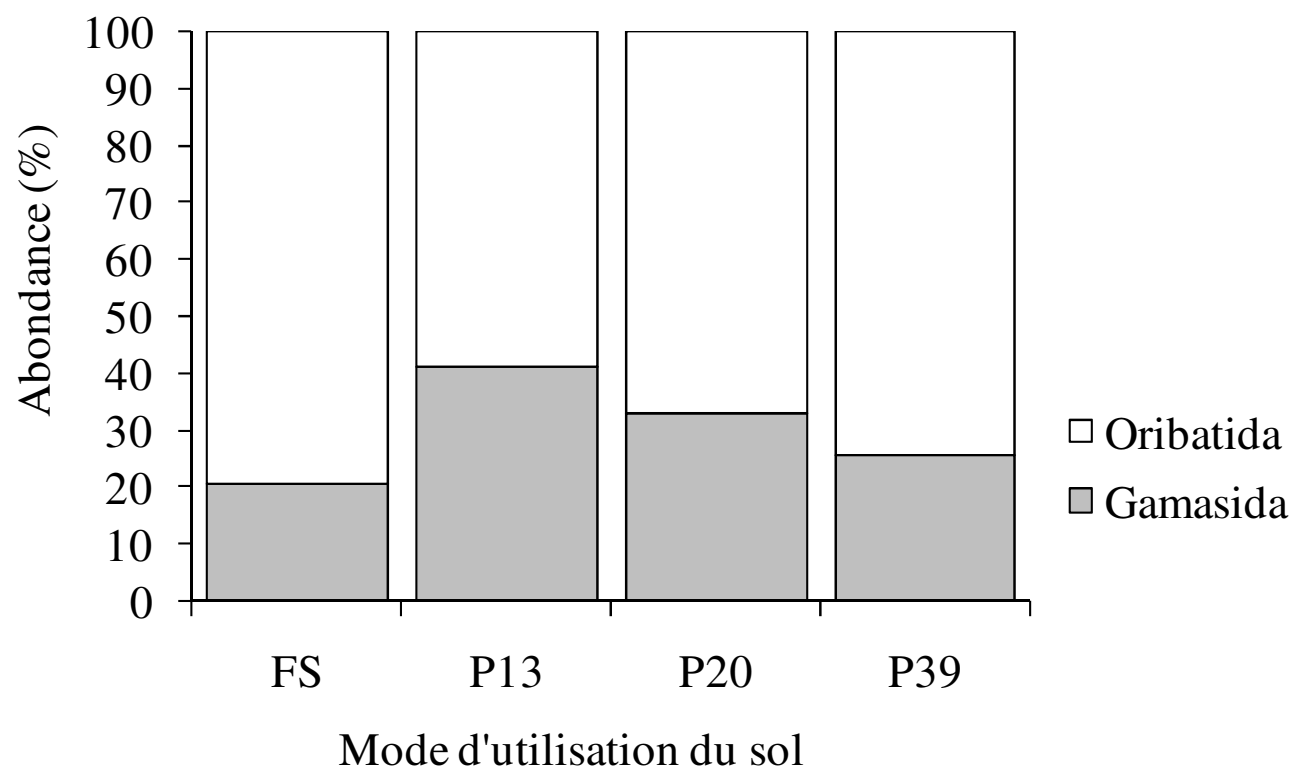

Figure 2 : Abondance relative (\%) des grands groupes d'Acariens. FS-Forêts secondaires, P13-Plantations de palmiers âgées de 13 ans, P20-Plantations de palmiers âgées de 20 ans, P39-Plantations de palmiers âgées de 39 ans.

Gamasides du sol: Sur l'ensemble des modes d'utilisation du sol, 19 espèces de Gamaside sont identifiées. $8,5,13$ et 10 espèces de Gamaside sont respectivement répertoriées dans la forêt secondaire et les plantations de palmiers âgées de 13, 20 et 39 ans. L'abondance des Gamasides diffèrent significativement 
(Anova 1, $F=2,88 ; P=0,043$ ) sur l'ensemble des modes d'utilisation du sol. Les Gamasina et les Uropodina constituent les deux grands groupes des Gamasides (Figure 3). L'abondance des Uropodina varie significativement (Anova 1, $F=3,09 ; P=0,034$ ) à travers les modes d'utilisation du sol. Leur abondance oscille entre $6 \pm 0,16$ individus (plantation de palmiers âgées de 13 ans) et $34 \pm 0,80$ individus (forêt secondaire). D'un mode d'utilisation du sol à un autre, l'abondance des Gamasina n'est pas significativement modifiée (Anova 1, $F=2,20 ; P=0,098)$. Toutefois, leur abondance varie entre $1 \pm 0,07$ individus (plantation de palmiers âgées de 13 ans) et $11 \pm 0,34$ individus (plantation de palmiers âgées de 39 ans). L'abondance des Uropodina et des Gamasina augmente avec l'âge croissant des plantations de palmiers.

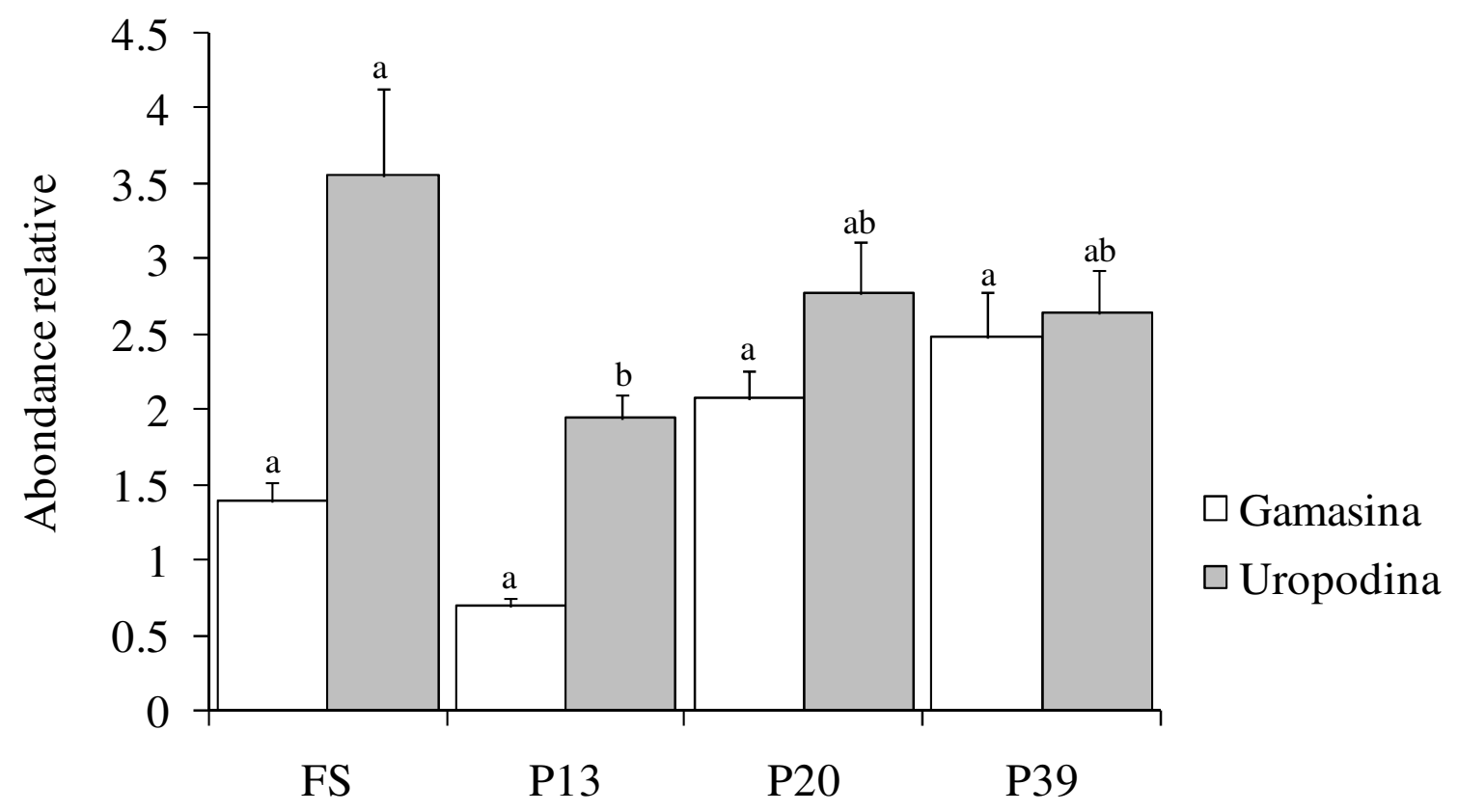

\section{Mode d'utilisation du sol}

Figure 3: Transformation logarithmique de l'abondance des sous-groupes de Gamasides à travers les modes d'utilisation du sol. FS-Forêts secondaires, P13-Plantations de palmiers âgées de 13 ans, P20-Plantations de palmiers âgées de 20 ans, P39-Plantations de palmiers âgées de 39 ans. $N=60$, Test d'Anova 1 ; les histogrammes identiques portant les mêmes lettres ne sont pas significativement différents au seuil de $5 \%$ (test de comparaison multiple de Tukey).

Oribates du sol: 49 espèces d'Oribates sont enregistrées sur l'ensemble des modes d'utilisation du sol. $31,9,19$ et 21 espèces d'Oribates sont respectivement identifiées dans la forêt secondaire et les plantations de palmiers âgées de 13, 20 et 39 ans. L'abondance des Oribates ne diffère pas significativement (Anova 1, $F=1,44 ; P=0,241$ ) sur l'ensemble des modes d'utilisation du sol. Les Mixonomata, les Desmonomata et les Brachypylina constituent les trois grands groupes d'Oribates (Figure 4). L'abondance des Mixonomata (Anova 1, $F=1,46 ; P=0,234$ ), Desmonomata (Anova 1, $\mathrm{F}=0,28 ; \mathrm{P}=0,842$ ) et des Brachypylina (Anova 1, $\mathrm{F}=$ $1,43 ; P=0,244)$ ne varie pas significativement sur l'ensemble des modes d'utilisation du sol. Les Brachypylina constituent l'essentiel des Oribates avec un maximum d'abondance observé en forêt secondaire (137 $\pm 6,57$ individus). L'abondance des Brachypylina augmente avec l'âge croissant des plantations de palmiers. Les Oribates supérieurs représentent plus de $90 \%$ de l'ensemble des Oribates quel que soit le mode d'utilisation du sol. Le ratio Oribate inférieur/Poronota n'excède pas 0,25 . L'abondance relative des Poronota représente $80 \%, 40 \%, 29 \%$ et $18 \%$ des Oribates respectivement dans la forêt secondaire et les plantations de palmiers âgées de 13, 20 et 39 ans. 


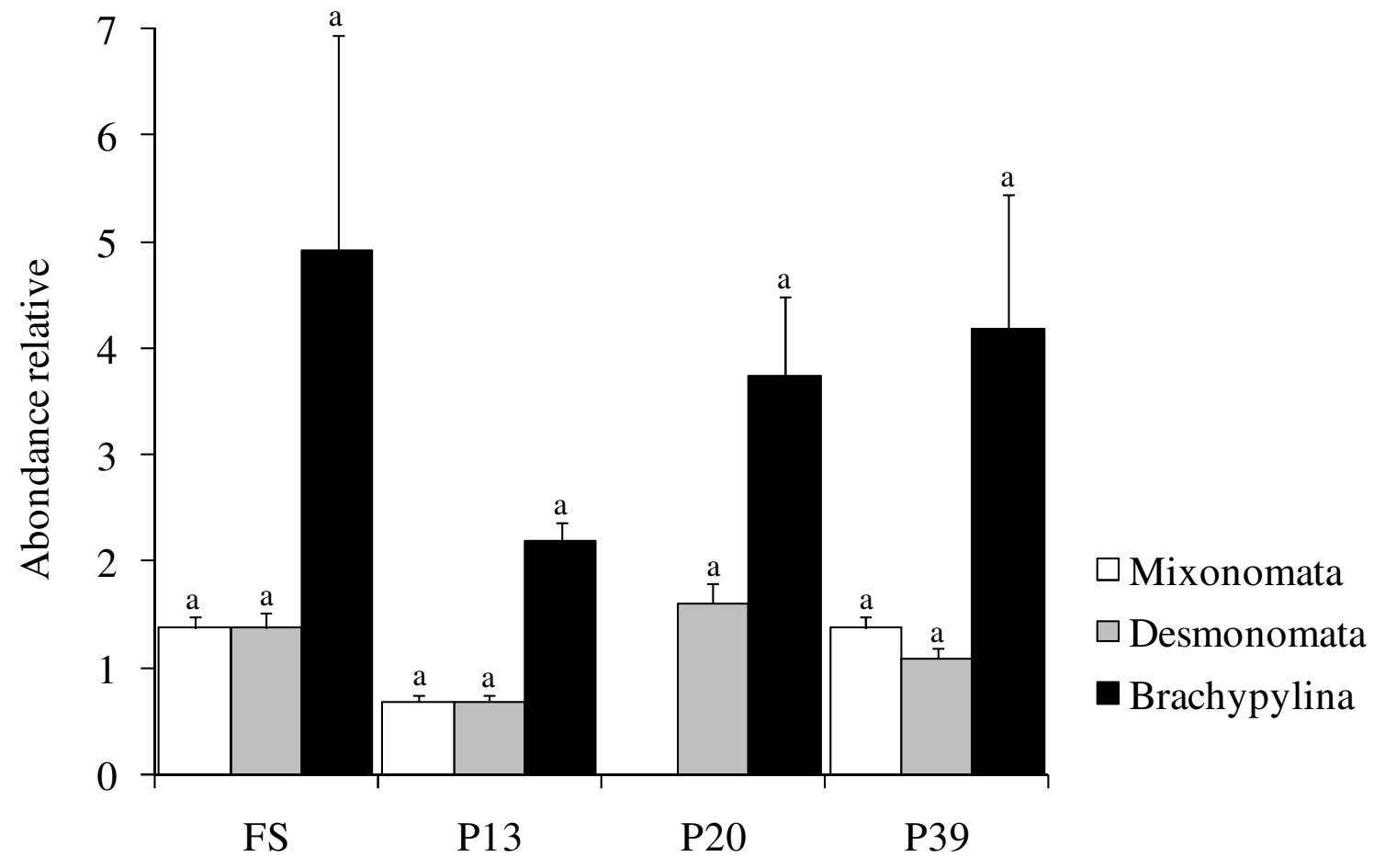

\section{Mode d'utilisation du sol}

Figure 4 : Transformation logarithmique de l'abondance des sous-groupes d'Oribates à travers les modes d'utilisation du sol. FS-Forêts secondaires, P13-Plantations de palmiers âgées de 13 ans, P20-Plantations de palmiers âgées de 20 ans, P39Plantations de palmiers âgées de 39 ans. $N=60$, Test d'Anova 1 ; les histogrammes identiques portant les mêmes lettres ne sont pas significativement différents au seuil de $5 \%$ (test de comparaison multiple de Tukey).

Indice de spécialisation de la communauté : L'analyse des valeurs des différentes dominances montre que 26 espèces rares et une espèce eudominante sont observées uniquement en forêt secondaire. Les espèces dominantes ( 3,2 et 3 espèces) et sous-dominantes (11, 2 et 3 espèces) sont respectivement observées dans les plantations de palmiers âgées de 13,20 et 39 ans (Tableau 5). 12 à 28 espèces mineures sont enregistrées à travers les modes d'utilisation du sol. 35 espèces spécialistes et 3 espèces généralistes sont répertoriées sur l'ensemble des modes d'utilisation du sol (voir Tableau S1). L'indice de spécialisation taxonomique indique que les Desmonomata et les Mixonomata sont à prédominance spécialistes tandis que les Uropodina présente une tendance généraliste (Tableau 6). Les Brachypylina et les Gamasina relatent une spécialisation intermédiaire. D'une manière générale, l'indice de spécialisation de la communauté estimé à l'échelle de la chronoséquence révèle une spécialisation intermédiaire. 
Tableau S1: Abondance (A) et fréquence (D) des espèces d'Acariens répertoriées sur les modes d'utilisation du sol. $\left({ }^{* *}\right)$ Espèces généralistes, $\left({ }^{*}\right)$ Espèces spécialistes. FS-Forêts secondaires, P13-Plantations de palmiers âgées de 13 ans, P20-Plantations de palmiers âgées de 20 ans, P39-Plantations de palmiers âgées de 39 ans. $\mathrm{N}=60$.

\begin{tabular}{|c|c|c|c|c|c|c|c|c|}
\hline \multirow[b]{3}{*}{ Morphoespèces } & \multicolumn{8}{|c|}{ Mode d'utilisation du sol } \\
\hline & \multicolumn{2}{|c|}{ FS } & \multicolumn{2}{|c|}{ P13 } & \multicolumn{2}{|c|}{ P20 } & \multicolumn{2}{|c|}{ P39 } \\
\hline & $A$ & $\mathbf{D}$ & A & D & A & D & A & D \\
\hline \multicolumn{9}{|l|}{ Gamasida } \\
\hline \multicolumn{9}{|l|}{ Gamasina } \\
\hline Eviphididae sp.1 & 0 & 0.00 & 0 & 0.00 & 2 & 2.99 & 5 & 5.32 \\
\hline Eviphididae sp.2* & 0 & 0.00 & 0 & 0.00 & 2 & 2.99 & 0 & 0.00 \\
\hline Eviphididae sp.3** & 3 & 1.67 & 1 & 5.88 & 1 & 1.49 & 5 & 5.32 \\
\hline Laelaptonyssidae sp. 1* $^{*}$ & 0 & 0.00 & 0 & 0.00 & 1 & 1.49 & 0 & 0.00 \\
\hline Pachylaelapidae sp.1 & 0 & 0.00 & 0 & 0.00 & 1 & 1.49 & 1 & 1.06 \\
\hline \multicolumn{9}{|l|}{ Uropodina } \\
\hline Afrotrachytes sp. $1^{\star *}$ & 9 & 5.00 & 2 & 11.76 & 1 & 1.49 & 4 & 4.26 \\
\hline Afrotrachytes sp. $2^{*}$ & 0 & 0.00 & 0 & 0.00 & 3 & 4.48 & 0 & 0.00 \\
\hline Uropodidae sp. $1^{*}$ & 5 & 2.78 & 0 & 0.00 & 0 & 0.00 & 0 & 0.00 \\
\hline Uropodidae sp.2 & 9 & 5.00 & 0 & 0.00 & 3 & 4.48 & 1 & 1.06 \\
\hline Uropodidae sp.5 & 4 & 2.22 & 0 & 0.00 & 1 & 1.49 & 0 & 0.00 \\
\hline Uropodidae sp. $6^{*}$ & 1 & 0.56 & 0 & 0.00 & 0 & 0.00 & 0 & 0.00 \\
\hline Uropodidae sp. $7^{\star}$ & 1 & 0.56 & 0 & 0.00 & 0 & 0.00 & 0 & 0.00 \\
\hline Evimirus uropodinus* & 0 & 0.00 & 0 & 0.00 & 1 & 1.49 & 0 & 0.00 \\
\hline Dinychidae sp.2 & 5 & 2.78 & 0 & 0.00 & 3 & 4.48 & 3 & 3.19 \\
\hline Trachyuropodidae sp.1 & 0 & 0.00 & 2 & 11.76 & 2 & 2.99 & 0 & 0.00 \\
\hline Gamasida sp.1 & 0 & 0.00 & 0 & 0.00 & 1 & 1.49 & 1 & 1.06 \\
\hline Gamasida sp.2 & 0 & 0.00 & 1 & 5.88 & 0 & 0.00 & 1 & 1.06 \\
\hline Gamasida sp.3 & 0 & 0.00 & 1 & 5.88 & 0 & 0.00 & 2 & 2.13 \\
\hline Gamasida sp. $4^{*}$ & 0 & 0.00 & 0 & 0.00 & 0 & 0.00 & 1 & 1.06 \\
\hline \multicolumn{9}{|l|}{ Oribatida } \\
\hline \multicolumn{9}{|l|}{ Mixonomata } \\
\hline Phthiracarus sp.1* & 1 & 0.56 & 0 & 0.00 & 0 & 0.00 & 0 & 0.00 \\
\hline Meristacarus sp.2 & 1 & 0.56 & 0 & 0.00 & 0 & 0.00 & 1 & 1.06 \\
\hline Meristacarus sp.3* & 0 & 0.00 & 0 & 0.00 & 0 & 0.00 & 1 & 1.06 \\
\hline Javacarus sp. $1^{*}$ & 0 & 0.00 & 0 & 0.00 & 0 & 0.00 & 1 & 1.06 \\
\hline Annectacarus sp. $1^{*}$ & 1 & 0.56 & 0 & 0.00 & 0 & 0.00 & 0 & 0.00 \\
\hline
\end{tabular}


Ahui et al., J. Appl. Biosci. 2018 Impact des plantations de palmier à huile conditions environnementales et du temps sur la structure des communautés d'Acariens du sol de la station de La Mé, Côte d'Ivoire

Eulohmannia sp. 1* $^{*}$

Desmonomata

Nothrus sp. $2^{*}$

Nothrus sp. $4^{*}$

Nothrus sp. 6

Nothrus sp. $7^{*}$

Afronothrus sp.1

Malaconothrus sp. $1^{*}$

Brachypylina

Dolicheremaeus sp.1

Lopheremaeus sp.1

Paralopheremaeus sp. 1 $^{\text {* }}$

Damaeidae sp.1

Damaeidae sp.3

Oppia sp.1*

Oppia sp.6

Oppia sp.13

Oppia sp. 15

Oppia sp.16

Oppia sp. $17^{\star}$

Oppia sp.18*

Oppiidae sp.4

Oppiidae sp.6

Neoliodes sp. 1

Eremobelba sp. ${ }^{*}$

Carabodidae sp. $5^{\star}$

Brachypylina, Poronota

Ceratozetidae sp.1

Ceratozetidae sp. 3

Ceratozetidae sp. $5^{*}$

Galumna sp.1

Galumna sp.2

Galumna sp.4*

Galumna sp. $5^{*}$

Galumna sp.11*

$\begin{array}{ll}0 & 0.00 \\ 0 & 0.00 \\ 0 & 0.00 \\ 0 & 0.00 \\ 0 & 0.00 \\ 3 & 1.67 \\ 0 & 0.00 \\ & \\ 1 & 0.56 \\ 0 & 0.00 \\ 0 & 0.00 \\ 2 & 1.11 \\ 0 & 0.00 \\ 0 & 0.00 \\ 1 & 0.56 \\ 0 & 0.00 \\ 5 & 2.78 \\ 1 & 0.56 \\ 1 & 0.56 \\ 0 & 0.00 \\ 3 & 1.67 \\ 5 & 2.78 \\ 1 & 0.56 \\ 1 & 0.56 \\ 1 & 0.56 \\ 1 & 0.56 \\ 0 & 0.00 \\ 0 & 0.00 \\ 1 & 0.56 \\ 1 & 0.56 \\ 1 & 0.56 \\ 1 & 0.56 \\ 2 & 1.11 \\ & \end{array}$

1

0.00

0.00

5.88

0.00

0.00

0.00

0.00

5.88

5.88

0.00

5.88

0.00

0.00

5.88

0.00

0.00

0.00

0.00

0.00

0.00

0.00

0.00

0.00

0.00

5.88

0.00

0.00

0.00

0.00

0.00

\begin{tabular}{|c|c|c|c|}
\hline 0 & 0.00 & 0 & 0.00 \\
\hline 0 & 0.00 & 1 & 1.06 \\
\hline 1 & 1.49 & 0 & 0.00 \\
\hline 1 & 1.49 & 0 & 0.00 \\
\hline 0 & 0.00 & 1 & 1.06 \\
\hline 1 & 1.49 & 0 & 0.00 \\
\hline 1 & 1.49 & 0 & 0.00 \\
\hline 1 & 1.49 & 2 & 2.13 \\
\hline 1 & 1.49 & 0 & 0.00 \\
\hline 0 & 0.00 & 0 & 0.00 \\
\hline 0 & 0.00 & 4 & 4.26 \\
\hline 1 & 1.49 & 0 & 0.00 \\
\hline 0 & 0.00 & 11 & 11.70 \\
\hline 6 & 8.96 & 2 & 2.13 \\
\hline 8 & 11.94 & 10 & 10.64 \\
\hline 1 & 1.49 & 7 & 7.45 \\
\hline 0 & 0.00 & 2 & 2.13 \\
\hline 0 & 0.00 & 0 & 0.00 \\
\hline 2 & 2.99 & 0 & 0.00 \\
\hline 7 & 10.45 & 12 & 12.77 \\
\hline 0 & 0.00 & 0 & 0.00 \\
\hline 1 & 1.49 & 2 & 2.13 \\
\hline 0 & 0.00 & 0 & 0.00 \\
\hline 0 & 0.00 & 0 & 0.00 \\
\hline 3 & 4.48 & 3 & 3.19 \\
\hline 1 & 1.49 & 2 & 2.13 \\
\hline 0 & 0.00 & 0 & 0.00 \\
\hline 0 & 0.00 & 1 & 1.06 \\
\hline 1 & 1.49 & 0 & 0.00 \\
\hline 0 & 0.00 & 0 & 0.00 \\
\hline 0 & 0.00 & 0 & 0.00 \\
\hline 0 & 0.00 & 0 & 0.00 \\
\hline
\end{tabular}


Ahui et al., J. Appl. Biosci. 2018 Impact des plantations de palmier à huile conditions environnementales et du temps sur la structure des communautés d'Acariens du sol de la station de La Mé, Côte d'Ivoire

Galumna sp.15*

Galumna sp.16

Galumnella sp. $1^{*}$

Orthogalumna sp.1*

Taeniogalumna sp. $1^{\star}$

Mycobatidae sp.2

Scheloribatidae sp. $3^{* *}$

Scheloribatidae sp. $4^{*}$

Haplozetidae sp.1

Acaridida sp.1

Rhizoglyphus sp. $1^{*}$

Oribatida sp. $1^{*}$

0.56
0.56
0.56
0.56
0.56
0.56
0.56
0.56
0.00
55.00
0.00
0.56

$\begin{array}{llllll}0 & 0.00 & 0 & 0.00 & 0 & 0.00 \\ 2 & 11.76 & 0 & 0.00 & 0 & 0.00 \\ 0 & 0.00 & 0 & 0.00 & 0 & 0.00 \\ 0 & 0.00 & 0 & 0.00 & 0 & 0.00 \\ 0 & 0.00 & 0 & 0.00 & 0 & 0.00 \\ 0 & 0.00 & 0 & 0.00 & 1 & 1.06 \\ 1 & 5.88 & 5 & 7.46 & 4 & 4.26 \\ 0 & 0.00 & 0 & 0.00 & 0 & 0.00 \\ 0 & 0.00 & 1 & 1.49 & 1 & 1.06 \\ 0 & 0.00 & 2 & 2.99 & 0 & 0.00 \\ 0 & 0.00 & 0 & 0.00 & 1 & 1.06 \\ 0 & 0.00 & 0 & 0.00 & 0 & 0.00\end{array}$

Tableau 5 : Proportion de dominance des espèces d'Acariens. FS-Forêts secondaires, P13-Plantations de palmiers âgées de 13 ans, P20-Plantations de palmiers âgées de 20 ans, P39-Plantations de palmiers âgées de 39 ans.

\begin{tabular}{lcccc}
\hline & \multicolumn{3}{c}{ Mode d'utilisation du sol } \\
\cline { 2 - 5 } Caractéristiques des espèces & FS & P13 & P20 & P39 \\
\hline Eudominantes $(>30 \%)$ & 1 & 0 & 0 & 0 \\
Dominantes (10-30\%) & 0 & 3 & 2 & 3 \\
Sous-dominantes (5-10\%) & 0 & 11 & 2 & 3 \\
Mineures (1-5\%) & 12 & 0 & 28 & 25 \\
Rares $(<1 \%)$ & 26 & 0 & 0 & 0 \\
\hline
\end{tabular}


Tableau 6 : Indice de spécialisation de la communauté d'Acariens observés le long de la chronoséquence de palmier à huile

\begin{tabular}{ccl}
\hline Groupes Majeurs & \multicolumn{2}{c}{ Indice de spécialisation taxonomique } \\
\hline Uropodina & 0,97 & Généraliste \\
Brachypylina & 1,00 & Intermédiaire \\
Gamasina & 1,81 & Intermédiaire \\
Desmonomata & 2,75 & Spécialiste \\
Mixonomata & 2,75 & Spécialiste \\
\hline Indice de spécialisation de la communauté & $1,85 \pm 0,40$ & Intermédiaire \\
\hline
\end{tabular}

Impact des propriétés physico-chimiques du sol sur les caractéristiques des Acariens édaphiques : Quel que soit le mode d'utilisation du sol, les propriétés physiques du sol n'impactent pas significativement la densité des Acariens. Cependant, la richesse spécifique des Acariens est significativement affectée par la densité apparente à travers les plantations de palmiers âgées de 13 ans. A part le pH et le carbone accumulé annuellement dans les plantations de palmiers âgées de 13-39 ans, ainsi que le long de la chronoséquence de palmier, les propriétés chimiques du sol gouvernent significativement la densité des Acariens, respectivement dans les plantations de palmiers âgées de 13, 39, 13-39, et le long de la chronoséquence (Tableau 7). Le carbone organique du sol et la matière organique du sol impactent significativement la richesse spécifique des Acariens, respectivement dans les plantations de palmiers âgées de $13,39,13-39$ ans, et le long de la chronoséquence. Le $\mathrm{pH}$ et le carbone accumulé annuellement affectent significativement la richesse spécifique, respectivement dans les plantations de palmiers âgées de 13 et 39 ans. Le stock de carbone et le carbone séquestré gouvernent significativement la richesse spécifique des Acariens dans les plantations de palmiers âgées de 39, 13-39 ans, et le long de la chronoséquence. 
Ahui et al., J. Appl. Biosci. 2018 Impact des plantations de palmier à huile conditions environnementales et du temps sur la structure des communautés d'Acariens du sol de la station de La Mé, Côte d'Ivoire

Tableau 7 : Interaction entre les paramètres biologiques des Acariens et les caractéristiques physico-chimiques du sol.

\begin{tabular}{|c|c|c|c|c|c|c|c|c|c|c|}
\hline \multicolumn{2}{|c|}{$P 13$} & \multicolumn{2}{|c|}{ P20 } & \multicolumn{2}{|c|}{ P39 } & \multicolumn{2}{|c|}{ P13-39 } & \multicolumn{2}{|c|}{ FS } & Chr \\
\hline$R$ & $P$ & $R$ & $P$ & $R$ & $P$ & $R$ & $P$ & $R$ & $P$ & $R$ \\
\hline
\end{tabular}

Densité

Densité apparente $\left(\mathrm{g}_{\mathrm{cm}} \mathrm{cm}^{-3}\right)$

Teneur en eau (\%)

Carbone organique du sol $\left(\mathrm{g} . \mathrm{kg}^{-1}\right.$ sol)

Matière organique du sol $\left(\mathrm{g}^{\mathrm{kg}} \mathrm{kg}^{-1} \mathrm{sol}\right)$

$\mathrm{pH}-\mathrm{H}_{2} \mathrm{O}$

Carbone accumulé (t.ha-1. $\left.\mathrm{an}^{-1}\right)$

Stock de carbone (t.ha-1)

$-0,45 \quad 0,216$

$0,34 \quad 0,369$

$0,30 \quad 0,422$

$0,03 \quad 0,880$

$\begin{array}{rr}0,03 & 0,880 \\ -0,33 & 0,085\end{array}$

$\begin{array}{ll}-0,25 & 0,500\end{array}$

$\begin{array}{ll}-0,07 & 0,677\end{array}$

$0,10 \quad 0,779$

$-0,65 \quad 0,055$

$-0,590,090$

$0,550,002^{* *}$

$0,31 \quad 0,404$

$\begin{array}{ll}-0,20 & 0,237\end{array}$

$\begin{array}{ll}0,69 & 0,038^{*} \\ 0,69 & 0,038^{*}\end{array}$

$0,14 \quad 0,702$

$0,73 \quad 0,024^{*}$

$0,550,002^{* *}$

$\begin{array}{ll}-0,25 & 0,500\end{array}$

$\begin{array}{ll}-0,25 & 0,500\end{array}$

$0,49 \quad 0,002^{\text {** }}$

$0,56 \quad 0112$

$\begin{array}{ll}0,14 & 0,702 \\ 0,08 & 0,823\end{array}$

$\begin{array}{ll}0,73 & 0,024^{*} \\ 0,37 & 0,320\end{array}$

$-0,08 \quad 0,665$

$0,30 \quad 0,430$

$0,490,002^{* *}$

$0,67 \quad 0,045^{*}$

$0,22 \quad 0,556$

$0,68 \quad 0,039^{*}$

$0,26 \quad 0,173$

$\begin{array}{ll}-0,42 \quad 0,251 \\ -0,42 & 0,251\end{array}$

$\begin{array}{ll}-0,05 & 0,729\end{array}$

Carbone séquestré (Teq $\mathrm{CO}_{2} \cdot$ ha $^{-1}$ )

$0,67 \quad 0,045^{*}$

$0,22 \quad 0,556$

$0,68 \quad 0,039^{*}$

$0,56 \quad 0,002^{* *}$

$\begin{array}{ll}-0,42 & 0,251\end{array}$

$-0,09 \quad 0,588$

Richesse spécifique

Densité apparente $\left(\mathrm{g} . \mathrm{cm}^{-3}\right)$

Teneur en eau (\%)

Carbone organique du sol (g.kg-1 sol)

Matière organique du sol $\left(\mathrm{g}^{\mathrm{kg}} \mathrm{kg}^{-1} \mathrm{sol}\right)$

$\mathrm{pH}-\mathrm{H}_{2} \mathrm{O}$

Carbone accumulé (t.ha-1. an $\left.^{-1}\right)$

Stock de carbone (t.ha-1)

$0,22 \quad 0,556$

$0,68 \quad 0,039^{*}$

$0,560,002^{* *}$

$-0,42 \quad 0,251$

$0,46 \quad 0,004^{* *}$

$0,46 \quad 0,004^{* *}$

Carbone séquestré ( $\mathrm{Teq} \mathrm{CO}_{2}$ ha $^{-1}$ )

$\begin{array}{cccc}-0,66 & 0,049^{*} & -0,03 & 0,929 \\ -0,03 & 0,918 & -0,34 & 0,363 \\ 0,80 & 0,008^{* *} & -0,22 & 0,561 \\ 0,80 & 0,008^{* *} & -0,22 & 0,561 \\ 0,77 & 0,013^{*} & 0,01 & 0,964 \\ 0,64 & 0,059 & -0,05 & 0,894 \\ 0,64 & 0,059 & -0,05 & 0,894 \\ 0,64 & 0,059 & -0,05 & 0,894\end{array}$

$0,23 \quad 0,542$

$-0,17 \quad 0,396$

$0,19 \quad 0,621$

$\begin{array}{ll}-0,04 & 0,911\end{array}$

$-0,17 \quad 0,315$

$\begin{array}{llll}-0,63 & 0,064 & -0,32 & 0,102\end{array}$

$\begin{array}{llll}0,84 & 0,004^{* *} & 0,42 & 0,028^{*}\end{array}$

$\begin{array}{ll}-0,41 & 0,263\end{array}$

$-0,27 \quad 0,098$

$0,840,004^{* *}$

$0,42 \quad 0,028^{*}$

$-0,41 \quad 0,263$

$0,39 \quad 0,017^{*}$

$\begin{array}{llll}0,47 & 0,201 & 0,11 & 0,551\end{array}$

$0,35 \quad 0,345$

$0,390,017^{*}$

$\begin{array}{llll}0,78 & 0,012^{*} & 0,03 & 0,867\end{array}$

$\begin{array}{ll}-0,33 & 0,384\end{array}$

$-0,21 \quad 0,209$

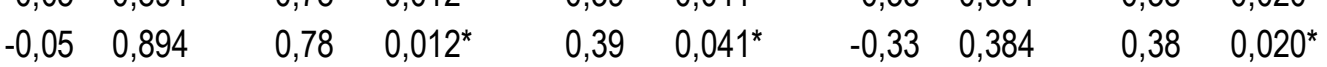

FS-Forêts secondaires, P13-Plantations de palmiers âgées de 13 ans, P20-Plantations de palmiers âgées de 20 ans, P39-Plantations de palmiers âgées de 39 ans, P13-P39Plantations de palmiers âgées de 13 à 39 ans, Chr-Chronoséquence. ${ }^{*} p<0,05 ;{ }^{* *} p<0,01$ 


\section{DISCUSSION}

La conversion des forêts tropicales en plantations de palmier à huile entraîne des pertes en biodiversité et par conséquent des services et fonctions éco-systémiques (Koh et Wilcove, 2008 ; Gérard, 2016 ; Ghazali et al., 2016 ; Tao et al., 2016). Cette observation est similaire aux résultats de la présente investigation. En effet, l'analyse des données révèle une baisse de la densité ($88 \%$ et $-48 \%$ ), richesse spécifique $(-64 \%$ et $-21 \%$ ) et de la diversité (-64\% et $-8 \%$ ), respectivement 13 ans et 39 ans après la conversion de la forêt secondaire en plantations de palmiers. Les travaux préparatoires (abattages d'anciens plants, défriches, sarclages) liés à la mise en place et à l'entretien des parcelles pourraient expliquer la faible valeur d'abondance et de richesse spécifique des Acariens observés sur l'ensemble des plantations (Bedano et al., 2005). En effet, les activités agricoles modifient potentiellement les propriétés du sol et altèrent la qualité biologique du milieu (Gbarakoro et Zabbey, 2013 ; Ghazali et al., 2016 ; Tao et al., 2016). La faible capacité des Oribates à échapper aux perturbations et à recoloniser les habitats fortement perturbés (Berthet, 1964) pourrait aussi expliquer la diminution drastique de l'abondance et de la diversité des Acariens dans les parcelles de palmiers âgées de 13 ans. A cela s'ajoutent les faibles teneurs en nutriments du sol. Le développement des Gamasides (Manu et Honciuc, 2010) et des Oribates (Hasegawa et al., 2012) est fortement influencé par le microclimat, qui varie suivant la structure des plantes herbacées, arbustes ou ligneux ainsi que la couche de la litière. Le sous-bois relativement dégagé et l'absence d'un réseau trophique assez diversifié limitent également la prolifération des Acariens dans les plantations âgées de 13 ans. Le cas inverse est observé en forêt secondaire, où la stabilité de l'environnement forestier (diversité des niches et de zones refuges) et la diversité des sources trophiques (litières et autres matières organiques mortes) consécutive à la diversité des ligneux contribueraient à maintenir un microclimat édaphique favorable au développement des Acariens $\mathrm{du}$ sol. Toutefois, avec la mise en place d'un système de gestion durable, les agrosystèmes peuvent fournir des conditions favorables pour la biodiversité du sol (Conti, 2015 ; Tao et al., 2016). Cette affirmation est soutenue par les résultats de cette investigation, d'autant plus que la richesse spécifique (39 espèces) et la diversité $(0,83)$ des Acariens en forêt secondaire demeurent relativement similaires à celles enregistrées dans les plantations de palmiers âgées de 39 ans (richesse spécifique: 31 espèces ; diversité : 0,76 ). Le maintien d'une couverture permanente du sol (légumineuse de couverture etc.) et des résidus de récolte dans les vieilles plantations de palmier à huile favorisent le rétablissement progressif des nutriments et la stabilité des fonctions écosystémiques (Conti, 2015 ; Tao et al., 2016). La similarité des valeurs des nutriments du sol mesurées dans la forêt secondaire et les plantations de palmiers âgées de 39 ans confirme cette approche explicative. La structure des communautés relate que la répartition des effectifs entre les diverses espèces reste presque similaire entre la forêt secondaire $(0,48)$ et les plantations de palmiers âgées de 39 ans $(0,50)$. Aussi, la dissimilarité estimée entre ces deux modes d'utilisations du sol $(57 \%)$ reste inférieure à celle enregistrée entre la forêt secondaire et les plantations de palmiers âgées de 13 ans (85\%). Néanmoins, l'identification de 35 espèces spécialistes et 3 espèces généralistes relatent l'existence d'une grande dissemblance dans la composition spécifique des communautés d'Acariens à travers la chronoséquence de palmier. Le dégrée d'interférence humaine dans les écosystèmes a toujours été un facteur déterminant dans la stabilité des communautés d'Acariens du sol (BehanPelletier, 1999 ; Yang et al., 2015). Les travaux réalisés par Yéo (2018) sur les mêmes parcelles de la station de La Mé a permis de déterminer les valeurs des indices de dégradation des sols $(0,01 ; 0,08 ; 0,05$ et 0,02$)$, issues respectivement de la forêt secondaire et des plantations de palmiers âgées de 13, 20 et 39 ans. En d'autres termes, la dégradation des sols diminue avec le vieillissement des plantations de palmiers. Le processus de restauration de la stabilité des sols, après la conversion de la forêt secondaire s'est caractérisé par une augmentation des composantes biologiques du sol (densité, richesse spécifique et diversité des Acariens, richesse spécifique des Oribates, Uropodina et des Brachypylina) suivant l'âge croissant des plantations. Toutefois, la variation en sens inverse de l'abondance relative des Oribates et des Gamasides le long des plantations âgées de 13, 20 et 39 ans s'expliquerait par le fait que la biomasse de ces deux grands groupes d'Acariens change inversement à mesure que les perturbations du sol augmentent dans les écosystèmes (Bedano et Ruf, 2006). Les Oribates émergent dans les écosystèmes relativement stables (Behan-Pelletier, 1999), tandis que les Gamasides prolifèrent plus facilement dans des écosystèmes instables (Manu, 2013). Les Oribates représenteraient 59 à $79 \%$ de l'ensemble des Acariens identifiés à travers la chronoséquence de palmiers. Une tendance similaire a 
été constatée au Japan, où Hasegawa et al. (2012) ont enregistré $75 \%$ et $83 \%$ d'Oribates, respectivement dans des chronoséquences de conifères et de forêts. Pareillement, 64 à $74 \%$ d'Oribates ont été observés le long d'une chronoséquence d'hévéa en Côte d'Ivoire (N'Dri et al., 2017). Ces fortes abondances d'Oribates seraient probablement dues à un effet saison sur les horizons de surface, caractérisé par une augmentation de l'abondance et de la diversité des Acariens du sol en saison pluvieuse (N'Dri et al., 2011). Le nombre élevé d'espèces rares (26 espèces) présents uniquement dans la forêt secondaire confirme l'hypothèse selon laquelle la conversion des forêts en plantations de palmier à huile $a$ un effet négatif sur les espèces d'Acariens peu abondants. Les espèces rares ont des caractéristiques écologiques plus originales que celles dites abondantes, soit une fonction singulière difficilement remplaçable en cas de disparition (Bertrand, 2013). La destruction continue de cette forêt au profit de l'expansion des plantations de palmier à huile pourrait engendrer l'extinction de ces espèces. En effet, les espèces rares d'aujourd'hui sont potentiellement les espèces abondantes de demain (Bertrand, 2013). L'effet d'échantillonnage et la complémentarité entre espèces sont les deux mécanismes permettant d'expliquer la relation positive entre la biodiversité et le fonctionnement

\section{CONCLUSION}

L'objectif de l'étude réalisée au sein de la station de La Mé consistait à évaluer les différentes modifications de la structure des communautés d'Acariens du sol observés à travers une chronoséquence de palmier à huile. Conformément à l'hypothèse préalablement établie, les résultats révèlent que la densité des Acariens (- $88 \%$ et $48 \%)$, la richesse spécifique (-64\% et $-21 \%$ ) et la diversité $(-64 \%$ et $-8 \%)$ ont diminué, respectivement, 13 ans et 39 ans après la conversion de la forêt secondaire en plantations de palmiers. Au total, 68 espèces d'Acariens ont été répertoriées le long des modes d'utilisation du sol. L'abondance relative des Oribates augmente avec l'âge croissant des plantations de palmiers tandis que la

\section{REMERCIEMENTS}

Les auteurs remercient infiniment la Direction administrative du Centre National de Recherche Agronomique de la Station de La Mé, pour avoir facilité l'accès aux parcelles d'études, ainsi que notre séjour durant les travaux de terrain. Un grand merci au Prof. TIHO Seydou, Doyen de I'Unité de Formation et de des écosystèmes (Hooper et al., 2005). En effet, lorsque le nombre d'espèces augmente, la probabilité qu'une espèce assurant la fonction étudiée soit présente augmente. La complémentarité des espèces leur permet d'utiliser différemment les ressources, limitant ainsi la compétition entre espèces et favorisant une meilleure productivité (Turcati, 2011). Les richesses spécifiques observées représentent 56 à $66 \%$ des richesses spécifiques attendues. Quel que soit le mode d'utilisation du sol, le plateau de saturation n'est pas atteint, indiquant un taux de recrutement relativement acceptable. La taille d'échantillonnage assez moyenne, le faible rendement du Berlese Tullgren et l'absence d'espèces rares et eudominantes dans les plantations de palmiers âgées de 13,20 et 39 ans pourraient expliquer la différence entre les richesses spécifiques observées et attendues. Après 39 ans de conversion de la forêt secondaire en plantations de palmiers, le processus de restauration de la qualité du sol n'a pas favorisé un changement significatif des composantes biologique $\mathrm{du}$ sol. Néanmoins, cette investigation souligne une amélioration de la qualité biologique du sol avec le temps, et qui se caractérise par une augmentation de la densité des Acariens $(+336 \%)$, richesse spécifique $(+121 \%)$ et de la diversité $(+153 \%)$, respectivement dans les plantations de palmiers âgées de 39 ans comparées à celles de 13 ans.

tendance inverse est observée avec les Gamasides. Les espèces rares et les espèces eudominantes caractérisent la forêt secondaire, pendant que les espèces dominantes et sous-dominantes prédominent dans les plantations de palmiers âgées de 13, 20 et 39 ans. L'étude révèle également une forte dissemblance dans la composition spécifique des communautés d'Acariens observées le long de la chronoséquence de palmiers. L'écosystème forestier est plus favorable au développement des Acariens, cependant une tendance à la recolonisation des plantations serait plus importante au fur et à mesure que les plantations de palmiers vieillissent.

Recherche (UFR) des Sciences de la Nature pour avoir favorisé notre insertion à la Station de La Mé. Reconnaissances et amitiés sincères aux Doctorants N'DA Guy Arnaud Rodolphe et TANO Ekra Kouamé pour leur assistance durant les travaux de terrain. 


\section{RÉFÉRENCES BIBLIOGRAPHIQUES}

Acharya S, Basu P. and Majumder S, 2014. Comparative study of diversity of soil oribatid mites (Acari : Oribatida) in two different soil habitats near Kolkata, West Bengal, India. Glob. J. Sci. Front. Res. 14, 16-21.

Balogh J. and Balogh P, 1992. The Oribatid mites genera of the World (Vol. 1 and 2). The Hungarian National Museum Press, Budapest, Hungary.

Barrios E, 2007. Soil biota, ecosystem services and land productivity. Ecological Economics 64, 269-285.

Bedano JC. and Ruf A, 2006. Soil predatory mite communities (Acari: Gamasina) in agroecosystems of Central Argentina. Applied Soil Ecology 36, 22-31.

Bedano JC, Cantú MP. and Doucet ME, 2005. Abundance of soil mites (Arachnida : Acari) in a natural soil of central Argentina. Zoological Studies 44, 505-512.

Behan-Pelletier VM, 1999. Oribatid mite biodiversity in agroecosystems : role for bioindication. Agric. Ecosyst. Environ. 74, 411-423.

Berthet PL, 1964. Field Study of the Mobility of Oribatei (Acari), Using Radioactive Tagging. The Journal of Animal Ecology 33, 443-449.

Bertrand MN, 2013. À espèce rare, fonction écologique unique. (Consulté le 19/02/2018) https://humanite.fr/environnement/espece-rarefonction-ecologique-unique-542716

Brou Yao T, Oszwald J, Bigot S. et Dervat E, 2005. Risques de Déforestation dans le Domaine Permanent de l'État de Côte d'Ivoire. Quel avenir pour ces derniers massifs Forestiers ? Télédétection 5, 121-205.

Carrère R, 2010. Le palmier à huile en Afrique : le passé, le présent et le futur. Collection du WRM sur les plantations №15, 69p.

Carron MP, Auriac Q, Snoeck D, Villenave C, Blanchart $E$, Ribeyre F, Marichal R, Darminto M. and Caliman JP, 2015. Spatial heterogeneity of soil quality around mature oil palms receiving mineral fertilization. European Journal of Soil Biology 66, 24-31.

Cheyns E, Akindès F. et Aka Adié F, 2000. La filière palmier à huile en Côte d'Ivoire 3 ans après la privatisation: état des lieux d'un procès de recomposition institutionnelle. Oléagineux, Corps Gras, Lipides 7(2), 166-171.
Coleman DC, Crossley Jr DA. and Hendrix PF, 2004. Fundamentals of soil ecology (2nd ed.). Academic Press, Burlington, U.S.A.

Colwell RK, 2000. Statistical Estimate of species Richness and Shared from Samples. Version 7.5. http://viceroy.eeb.uconn.edu/estimates (Consulté le 03/12/2017)

Conti FD, 2015. Conservation Agriculture and Soil Fauna : Only Benefits or also Potential Threats ? A review. E C Agriculture 2.5, 473-482.

Čuchta P. and Starý J, 2015. Preliminary results of a study on the soil mesofauna in disturbed spruce forest stands near Čertovo and Plešné Lakes in the Bohemian Forest (Czech Republic). Acta Soc. Zool. Bohem. 79, 161-167.

Drescher J, Rembold K, Allen K, Beckschäfer P, Buchori $D$, Clough Y, Faust H, Fauzi AM, Gunawan D, Hertel D, Irawan B, Jaya INS, Klamer B, Kleinn C, Knohl A, Kotowska MM, Krashevska V, Krishna V, Leuschner C, Lorenz W, Meijide A, Melati D, Nomura M, Pérez-Cruzado C, Qaim M, Siregar IZ, Steinebach S, Tjoa A, Tscharntke T, Wick B, Wiegand K, Kreft H. and Scheu S, 2016. Ecological and socio-economic functions across tropical land use systems after rainforest conversion. Phil. Trans. R. Soc. B. http://dx.doi.org/10.1098/rstb.2015.0275

Felton A, Gustafsson L, Roberge J-M, Ranius T, Hjältén J, Rudolphi J, Lindbladh M, Weslien J, Rist L, Brunet J. and Felton AM, 2016. How climate change adaptation and mitigation strategies can threaten or enhance the biodiversity of production forests: Insights from Sweden. Biological Conservation 194, 11-20.

Gbarakoro TN. and Zabbey N, 2013. Soil mesofauna diversity and responses to agro-herbicide toxicities in rainforest zone of the Niger Delta, Nigeria. Applied Journal of Hygiene 2, 01-07.

Gérard A, 2016. Experimental biodiversity enrichment in an oil-palm plantation. Frontiers in Plant Science 7, 15-38.

Gérard G. and Berthet P, 1966. A statistical study of microdistribution of Oribatei (Acari) Part II : The transformation of the data. Oikos 17, 142-149.

Ghazali A, Asmaha S, Syafiq M, Yahya MS, Aziz N, Peng $T L$, Norhisham AR, Puan CL, Turner EC. and Azhar B, 2016. Effects of monoculture and 
polyculture farming in oil palm smallholdings on terrestrial arthropod diversity. Journal of AsiaPacific Entomology 19, 415-421.

Hasegawa M, Okabe K, Fukuyama K, Makino S, Okochi I, Tanaka H, Goto H, Mizoguchi T. and Sakata T, 2012. Community structures of Mesostigmata, Prostigmata and Oribatida in broad-leaved regeneration forests and conifer plantations of various ages. Experimental and Applied Acarology 56, 4-21.

Hooper DU, Chapin FS, Ewel JJ, Hector A, Inchausti P, Lavorel S, Lawton JH, Lodge DM, Loreau M, Naeem S, Schmid B, Setälä H, Symstad AJ, Vandermeer J. and Wardle DA, 2005. Effects of biodiversity on ecosystem functioning: a consensus of current knowledge. Ecol. Monogr. 75, 3-35.

IPCC, 2001. Climate change 2001 : the scientific basis. Contribution of working group I to the IPCC. Third assessment report. Cambridge university press, Cambridge.

Jacquemard J-C, 2011. Le palmier à huile. Éditions Quæ, Versailles Cedex, France.

Jannot C, 2010. Emplois, économie, environnement : le développement de la filière palmier à huile en Côte-d'Ivoire. Oléagineux, Corps Gras, Lipides 17(6), 393-399.

Julliard R, Clavel J, Devictor V, Jiguet F. and Couvet D, 2006. Spatial segregation of specialists and generalists in bird communities. Ecology Letters 9, 1237-1244.

Kangah A. et Koli-Bi Z, 2015. Dynamique et transformation spatiale du littoral ivoirien : cas du littoral Alladjan, à l'Ouest d'Abidjan. Revue de Géographie Tropicale et d'Environnement 1, 2539.

Keenan RJ, Reams GA, Achard F, De Freitas JV, Grainger $A$. and Lindquist $E, 2015$. Dynamics of global forest area : Results from the FAO Global Forest Resources Assessment 2015. For. Ecol. Manage. 352, 9-20.

Koh LP. and Wilcove DS, 2008. Is oil palm agriculture really destroying tropical biodiversity? Conservation Letters 1, 60-64.

Krantz GW. and Walter DE, 2009. A Manual of Acarology (3rd ed.). Texas Tech., University Press, Lubbock, USA.

Lorber B, 2017. Les acariens. Institut de Biologie Moléculaire et Cellulaire du CNRS, Strasbourg, France.
Manu M, 2013. Diversity of soil mites (Acari : Mesostigmata, Gamasina) in various deciduous forest ecosystems of Muntenia region (southern Romania). Biological Lett 50, 3-16.

Manu M. and Honciuc V, 2010. Rank correlations at the level of soil mites (Acari : Gamasida ; Oribatida) from Central Parks of Bucharest city, Romania. Acta Entomol. Serbica 15(1), 129-140.

N'Dri JK, Andre HM. and Hance T, 2011. Soil mite diversity from Ivory Coast. European Journal of Scientific Research 64, 263-276.

N'Dri JK, Seka FA, Pokou PK, N'Da RAG. and Lagerlöf J, 2017. Abundance and diversity of soil mite (Acari) communities after conversion of tropical secondary forest into rubber plantations in Grand-Lahou, Côte d'Ivoire. Ecological Research 32, 909-919.

Palmafrique, 2017. Le palmier à huile dans l'économie Ivoirienne. (Consulté le 29/12/2017) http://www.palmafrique.com/lhuile-de-palmedans-leconomie-ivoirienne/

Paramananthan S, 2013. Soil properties and their influence on oil palm management and yield $I n$ : Webb MJ, Nelson PN, Bessou C, Caliman J-P. and Sutarta ES. Sustainable Management of Soil in Oil Palm Plantings. Aciar, pp.10-14.

Rafflegeau S, 2008. Dynamique d'implantation et conduite technique des plantations villageoises de palmier à huile au Cameroun: facteurs limitants et raisons des pratiques. Mémoire de doctorat en Agronomie, Institut des Sciences et Industries du Vivant et de l'Environnement (Agro Paris Tech), Centre de Recherches sur le Palmier à Huile de La Dibamba, École Doctorale ABIES, Cameroun, 149p.

Sangaré A, Koffi E, Akamou F. and Fall CA, 2009. Rapport national sur l'état des ressources phytogénétiques pour l'alimentation et l'agriculture. République de Côte d'Ivoire, 64p.

Scriven SA, Hodgson JA, McClean CJ. and Hill JK, 2015. Protected areas in Borneo may fail to conserve tropical forest biodiversity under climate change. Biological Conservation 184, 414-423.

Singh LA. and Ray DC, 2015. Effect of no-tillage and tillage on the ecology of mite, (Acarina: Oribatida) in two different farming systems of paddy field in Cachar district of Assam. Journal of Environmental Biology 36, 319-333.

Tao H-H, Slade EM, Willis KJ, Caliman J-P. and Snaddon $\mathrm{JL}, 2016$. Effects of soil management practices 
on soil fauna feeding activity in an Indonesian oil palm plantation. Agric. Ecosyst. Environ. 218, 133-140.

Thompson I, 2011. Biodiversité, seuils de tolérance des écosystèmes, résilience et dégradation des forêts. Unasylva $23862,25-30$.

Traoré K. et Péné CB, 2009. Étude Phytoécologique des Adventices dans les agroécosystèmes élaeicoles de La Mé et de Dabou. Journal of Applied Biosciences 104, 10005-10018.

Turcati L, 2011. Mesurer la biodiversité pour comprendre l'effet des perturbations sur les communautés végétales: apport des caractéristiques écologiques et évolutives des espèces. Mémoire de thèse de doctorat, Université Pierre et Marie Curie, France.

Walker RL, Wardle AD, Bardgett DR. and Clarkson DB, 2010. The use of chronosequences studies of ecological succession and soil development. Journal of Ecology 98, 725-736.

Walter DE, Latonas S. and Byers K, 2013. Almanac of Alberta Oribatida. Part 1. Ver. 2. 3. The Royal Aberta Museum, Edmonton, AB. http://www.royalabertamuseum.ca/natura/insects /reasearch/reasearch.htm

Yang B, Liu X, Ge F, Bao W, Fu S. and Liang W, 2015. Do shifts in soil Oribatida (Acari, Oribatida) give information on differences in fruit yield of Chinese star anise? Agriculture Ecosystems and Environment 207, 211-217.

Yéo JG, 2018. Étude de la variabilité des macroinvertébrés du sol et de la litière selon un gradient d'âge dans les plantations de palmier à huile de la station Irho La Mé. Mémoire de Master II, UFR Sciences de la Nature, Université Nangui Abrogoua, Côte d'Ivoire. 